\title{
The Influence of
}

\section{Dissolved Oxygen Concentration on the Respiration and Glucose Metabolism of Klebsiella aerogenes during Growth}

\author{
By D. E. F. HARRISON AND S. J. PIRT \\ Department of Microbiology, Queen Elizabeth College, London, W.8
}

(Accepted for publication 25 August 1966)

\section{SUMMARY}

The influence of dissolved oxygen concentration on the metabolism and respiration of growing Klebsiella aerogenes NCTC 8017 was studied by means of a continuous-flow culture technique. Different dissolved oxygen tensions (equivalent partial pressures) were obtained by varying the partial pressure of oxygen in the gas phase. The respiration rate (oxygen uptake rate per unit mass organism) was independent of dissolved oxygen tensions above $10-15 \mathrm{~mm}$. Hg when the culture was said to be in the 'excess oxygen state'. With dissolved oxygen tensions below about $10 \mathrm{~mm}$. $\mathrm{Hg}$, the 'limited oxygen state' developed. In the transition to this state, complex oscillations in the dissolved oxygen tension occurred. The oscillations reflected alternate stimulation and inhibition of the respiration rate. A decrease in dissolved oxygen tension to below $5 \mathrm{~mm}$. $\mathrm{Hg}$ immediately increased the respiration rate; an increase in dissolved oxygen tension from 5 to $10 \mathrm{~mm}$. $\mathrm{Hg}$ immediately decreased the respiration rate. These effects formed part of the mechanism of the oscillations which could be sustained indefinitely. The oscillations in respiration rate occurred irrespective of change from $\mathrm{pH} 6.0$ to 7.4 or whether growth was limited by glucose or ammonium supply. The pattern of oscillations in respiration rate varied with growth rate over the range $0 \cdot 2-0.5 \mathrm{hr}^{-1}$. It is suggested that decreasing the oxygen tension to about $5 \mathrm{~mm}$. $\mathrm{Hg}$ acted like an uncoupler of oxidative phosphorylation.

In the excess oxygen state with excess glucose and growth limited by ammonium supply, the glucose carbon was largely accounted for as $\mathrm{CO}_{2}$, pyruvate and organisms, irrespective of the $\mathrm{pH}$ value. Pyruvate was not accumulated when growth was glucose-limited. In the limited oxygen state, the glucose carbon was largely accounted for as: organisms, $\mathrm{CO}_{2}, 2: 3$-butanediol, ethanol, acetic acid, formic acid, lactic acid. The proportions of these fermentation products varied with oxygen supply, $\mathrm{pH}$ value and whether growth was ammonium-limited or glucose-limited.

\section{INTRODUCTION}

Studies on the influence of oxygen on growth and metabolism have been severely limited by the lack of a convenient and reliable means for the measurement of dissolved oxygen concentration. But the recent development of oxygen electrodes has made measurement of dissolved oxygen concentration over long periods much more feasible. In the present work, oxygen electrodes were used in the type of continuousflow culture referred to as a chemostat (Herbert, 1958). By this method it should be 
possible to maintain a constant dissolved oxygen concentration with a constant rate of organism growth provided the oxygen supply rate is constant. The relation of the dissolved oxygen concentration to the oxygen uptake rate and oxygen supply rate is given below. The oxygen electrode responds to oxygen activity or oxygen tension rather than to oxygen concentration (Kinsey \& Bottomley, 1963). The tension $(T)$ and concentration $(C)$ are related by the expression $T=f C$, where $f$ is an activity coefficient. Consequently the electrode readings are expressed in terms of equivalent oxygen partial pressure or tension; convenient units are mm. $\mathrm{Hg}$.

Most measurements of dissolved oxygen tension in cultures have been concerned with the effect of oxygen tension on respiration rate (e.g. Baumberger, 1939; Winzler, 1941; Longmuir, 1954; Chain \& Gaulandi, 1954; Chance, 1957; Phillips \& Johnson, 1961). The results of these and other workers generally indicate that respiration rate is independent of the dissolved oxygen tension until it reaches values in the range 0-15 mm. $\mathrm{Hg}$. There is a considerable variation in the value of the 'critical $\mathrm{O}_{2}$ tension'; that is, the value at which the respiration rate $\left(q_{\mathrm{O}_{2}}\right)$ begins to decrease; for the same organism, yeast, values from $0 \mathrm{~mm}$. $\mathrm{Hg}$ (Baumberger, 1939) to $12 \mathrm{~mm}$. $\mathrm{Hg}$ (Hixon \& Gaden, 1950) have been reported. The influence of growth rate on $q_{\mathrm{O}_{2}}$ has not been taken into account; however, it needs to be, because, as the data of Herbert (1958) showed, the $q_{\mathrm{O}_{2}}$ of bacteria increases linearly with their growth rate.

The existence of qualitative differences between the metabolic response of growing (multiplying) and non-growing bacteria to oxygen tension may be deduced from the observations on the adaptive response of enzyme systems to changes in oxygen supply. Several workers (e.g. Chin, 1950; Ephrussi, Slonimski \& Perrodin, 1950; Schaeffer, 1950) have shown the adaptive development of cytochromes in cells in response to access of oxygen. Moss (1952, 1956) investigated the relation between adaptive formation of cytochromes and dissolved oxygen tension in a growing culture of Aerobacter aerogenes. He used a continuous culture technique to maintain growth and an oxygen electrode to measure dissolved oxygen tension. He found that with decrease in the dissolved oxygen tension the amount of cytochrome $a_{2}$ in the bacteria increased and reached a maximum at an oxygen tension of about $0.2 \mathrm{~mm}$. $\mathrm{Hg}$. This quantitative control over the cytochrome content exerted by the environment is reminiscent of the quantitative control over the enzyme content of bacteria observed by Tempest \& Herbert (1965), rather than 'all or none' induced synthesis. Facultative anaerobic bacteria provide a particularly interesting subject for the study of oxygen relationships because their metabolism undergoes a profound change with change from an aerobic to an anaerobic environment (Gray, Wimpenny \& Mossman, 1966). The facultative anaerobe Klebsiella (Aerobacter) aerogenes was chosen for the present study because the effect of oxygen supply on the glucose metabolism of the growing organism had already been extensively studied (Pirt, 1957; Pirt \& Callow, 1958a, 1959). These workers compared fully aerobic growth with oxygen-limited growth, but they had no means of measuring the dissolved tension. The present study relates the glucose metabolism of Klebsiella aerogenes NCIB 8017 to the dissolved oxygen tension and reveals some new aspects of the behaviour of the organism at low oxygen tensions. 


\section{METHODS}

Relation between oxygen supply rate, oxygen uptake rate and dissolved oxygen tension. The parameters which affect the oxygen concentration of a submerged culture may be expressed as follows. Let $C=$ concentration of dissolved oxygen; $C_{s}=$ saturation concentration of dissolved oxygen; $t=$ time; $(d C / d t)_{s}=$ oxygen solution rate, that is, the rate of oxygen transfer from the gas to the liquid phase; $A=$ area of interface between gas and liquid.

For the oxygen solution rate or supply rate we can write (see Finn, 1954).

$$
\left(\frac{d C}{d t}\right)_{s}=\Phi A\left(C_{s}-C\right)
$$

where $\Phi=$ a constant which depends on the aeration conditions.

Let $T_{L}$ and $T_{G}(\mathrm{~mm} . \mathrm{Hg})$ be the oxygen tensions in the liquid and gas phases respectively.

$$
\begin{aligned}
& \text { Then } T_{L}=f C \\
& \text { and } T_{\theta}=f C_{s},
\end{aligned}
$$

where $f$ is a constant for a given medium.

From (1), (2) and (3) we have,

where $K=\Phi A \mid f$.

$$
\left(\frac{d C}{d t}\right)_{s}=K\left(T_{\theta}-T_{L}\right)
$$

For a culture we can write,

that is,

(net rate of increase in dissolved oxygen) $=$ (rate of oxygen supply)

$$
+ \text { (rate of oxygen uptake); }
$$

$$
\frac{d C}{d t}=K\left(T_{\theta}-T_{L}\right)-N
$$

where $N=$ oxygen uptake rate. In the steady state of a chemostat culture $d C / d t=\mathrm{O}$, and then,

$$
T_{L}=T_{\theta}-N / K
$$

It follows from (7) that as long as $N$, the oxygen uptake rate of the culture, is constant, with constant aeration conditions (i.e. constant $K$ ) the plot of $T_{L}$ against $T_{\theta}$ will be a straight line with a slope of unity, and the line will cut the $T_{a}$ axis at a point equal to $N / K$.

In the experiments to be described here it was not possible to control independently the dissolved oxygen tension since the required apparatus (MacLennan \& Pirt, 1966) was developed subsequently. Different oxygen tensions $\left(T_{L}\right)$ were obtained by varying the oxygen tension in the gas phase $\left(T_{G}\right)$. This was done by mixing nitrogen with the air supply. The total gas flow rate and the stirring rate were kept constant so that the term $K$ in equation (7) should be constant. Under such conditions any departure from a linear relation between $T_{L}$ and $T_{G}$ must be ascribed to a change in the oxygen uptake rate, $N$. 
Dissolved oxygen measurement. Although polarographic techniques have been used for many years for the measurement of dissolved oxygen concentration in cell suspensions, at the beginning of the present work there was no oxygen electrode which was proved suitable for use over long periods (up to several weeks) as required in continuous cultures. Consequently we investigated several techniques for the purpose. The first electrode used in this work was the E.I.L. Bishop Electrode (E.I.L. Richmond, Surrey) described by Bishop (1959). The Bishop electrode was initially used with a 'Lectrona' oxygen analyser kindly supplied by the British Oxygen Company Limited, Medical Department, London, S.W.1. The Lectrona (Bracken, Glover \& Longmuir, 1960) differs from the normal system of measuring the diffusion current produced by the electrode in that the electrode is used as a capacitor which is given a charge, and the rate of discharge of the electrode by oxygen is then measured. This system was found to be less suitable than was the measurement of diffusion currents. The zero current was very high (equivalent to $10 \mathrm{~mm} . \mathrm{Hg}$ of $\mathrm{O}_{2}$ ) and the calibration drift over a few days was large. When used to measure diffusion currents the Bishop electrode had a very low zero reading (equivalent to $<1 \mathrm{~mm}$. $\mathrm{Hg}$ pressure of $\mathrm{O}_{2}$ ) but the calibration still drifted. However, this drift in calibration became slight after the electrode had been in use for 7 days, and after this period the electrode could be used with greater confidence but it was less sensitive. When the electrode was set up, left for a week, then calibrated and used in a fermentation lasting 2 weeks, the maximum relative error due to drift was estimated to be $\pm 5 \%$.

In the latter part of the present work a scaled-down version of the Mackereth oxygen electrode (Mackereth, 1964) was obtained from E.I.L. Richmond, Surrey. This electrode was adapted for insertion into a culture vessel by replacing the Perspex cap by a Perspex rod. The Mackereth electrode is much more sensitive than the Bishop electrode and the calibration was found to be stable for over $1000 \mathrm{hr}$ continuous use in air-saturated solutions. A straight-line response was obtained up to over $160 \mathrm{~mm}$. $\mathrm{Hg} \mathrm{O}_{2}$ (air-saturated solution), and the lower limit of sensitivity was about $0.2 \mathrm{~mm}$. $\mathrm{Hg}$ of $\mathrm{O}_{2}$.

Oxygen electrodes were sterilized by ethylene oxide according to the method used by Callow \& Pirt (1956) for pH electrodes.

Continuous culture apparatus. The continuous culture apparatus used was of the chemostat type, similar to that used by Pirt \& Callow (1958a). The 2-1. culture vessel was equipped with automatic $\mathrm{pH}$ control.

The percentage oxygen in the gas supply to the culture was varied by mixing nitrogen from a cylinder ('White-spot' nitrogen supplied by British Oxygen Company, Wembley, Middlesex) and air from a pressure line. The gas flow rates were adjusted by means of Flostats (G. A. Platon Limited, Croydon, Surrey), which would compensate automatically for any variation in back pressure in the culture vessel. It was necessary that the composition of the gas-mixture supplied to the culture should be maintained very constant. A Manostat pressure regulator (John Watson and Smith Limited, Leeds) was used to ensure that the air pressure applied to the Flostat was constant. The gas mixture was passed through a 1-1. mixing bottle to smooth out any small sudden variations in gas composition. The gas flow rates were measured by Rotameter flow meters (Rotameter Manufacturing Company, Croydon, Surrey).

Culture medium. The medium contained (amounts in g./1.): $\mathrm{MgSO}_{4} .7 \mathrm{H}_{2} \mathrm{O}, 0.20$; $\mathrm{ZnSO}_{4} .7 \mathrm{H}_{2} \mathrm{O}, 0.001 ; \mathrm{MnSO}_{4} .4 \mathrm{H}_{2} \mathrm{O}, 0.001 ; \mathrm{FeSO}_{4} .7 \mathrm{H}_{2} \mathrm{O}, 0.005 ; \mathrm{CuSO}_{4} .5 \mathrm{H}_{2} \mathrm{O}$, 
$0.001 ; \mathrm{CaCl}_{2} .6 \mathrm{H}_{2} \mathrm{O}, 0.005 ; \mathrm{EDTA}, 0.25 ; \mathrm{KH}_{2} \mathrm{PO}_{4}, 6.0 ; \mathrm{NaH}_{2} \mathrm{PO}_{4} .2 \mathrm{H}_{2} \mathrm{O}, 1.07$. In experiments where growth was ammonium (nitrogen)-limited, ammonium sulphate, $0.6 \mathrm{~g}$./1. was added. When glucose was the growth-limiting substance, ammonium sulphate, $2.5 \mathrm{~g}$. 11 ., was added to the medium. Glucose was fed to the culture separately in the amounts stated below. The reservoir glucose solution contained $200 \mathrm{~g} . / 1$. for the experiments in which growth was nitrogen-limited, and $15 \mathrm{~g}$./1. for experiments in which growth was glucose-limited. The salts solution was adjusted to $\mathrm{pH} 6.3$ with $\mathrm{NaOH}$ when the final value was to be $\mathrm{pH} 6 \cdot 0$; for experiments at $\mathrm{pH} 7 \cdot 4$ the salts solution was adjusted to $\mathrm{pH} 7 \cdot 0$. The final $\mathrm{pH}$ was achieved in the culture vessel by automatic addition of either $2 \mathrm{~N}-\mathrm{NaOH}$ or $2 \mathrm{~N}-\mathrm{H}_{2} \mathrm{SO}_{4}$. The trace metal salts were dissolved separately in the EDTA solution with constant addition of $\mathrm{NaOH}$ to keep the $\mathrm{pH}$ value about $7 \cdot 0$. The salt solutions, the ammonium sulphate and the glucose were each autoclaved separately at $121^{\circ}$ for $20 \mathrm{~min}$.

Foam control. The apparatus included means for the automatic addition of antifoam at regular intervals (Pirt \& Callow, 1958b). The antifoam used was polyglycol P200 (Dow Chemicals, R. W. Greef and Co. Ltd., London). Foaming occurred occasionally and with the appearance of foam the oxygen tension in the culture decreased rapidly. The addition of antifoam to the culture was equally undesirable as it was noted that at $\mathrm{pH} 6.0$ the addition of antifoam caused an increase in oxygen tension of about $10 \mathrm{~mm}$. $\mathrm{Hg}$, even when there was no foam present. When antifoam was added to a culture at an alkaline $\mathrm{pH}(7.4)$, however, there was a sudden decrease in oxygen tension (about $5 \mathrm{~mm} . \mathrm{Hg}$ ) followed by a slow increase back to the original reading. The results reported here were obtained at periods when the culture was not foaming and antifoam had not been added.

Inoculum. The organism used was Klebsiella aerogenes NCIB 8017, obtained from the National Collection of Industrial Bacteria, Torry Research Station, Aberdeen. A fresh ampoule of freeze-dried culture was used to inoculate a shake-flask containing $20 \mathrm{ml}$. medium. When maximum growth was obtained in the shake-flask it was used as an inoculum for the continuous culture.

Other cultural conditions. All experiments were done at $30 \pm 0 \cdot 5^{\circ} \mathrm{The} \mathrm{pH}$ value was controlled to $\pm 0.05 \mathrm{pH}$ units. On changing the conditions of culture, at least 4 volumes were allowed to flow through the culture vessel before samples were taken. At least two samples were taken for each steady state, one or more volumes being allowed to pass through the culture vessel between samples. Aeration was by the vortex method with a stirring rate of $1050 \mathrm{rev} / \mathrm{min}$. unless otherwise stated. The stirrer was a vaned disc of $65 \mathrm{~mm}$. diameter; vane height, $13 \mathrm{~mm}$. above and $4 \mathrm{~mm}$. below disc.

Treatment of samples. Samples were taken into a boiling tube standing in iced water to effect rapid cooling. The opacity of the sample was measured immediately. The organisms were removed by centrifugation and the supernatant fluid stored at $4^{\circ}$ with a trace of mercuric chloride (one drop of a saturated solution $/ 20 \mathrm{ml}$.) added as a preservative.

Analytical methods. The methods of determining dry weight of organism, glucose, butanediol, acetoin, ethanol, lactic acid, formic acid, and volatile acid, were as described by Pirt (1957) and Pirt \& Callow (1958a). Acetic acid was determined by subtracting the formic acid present from the total volatile acid. Pyruvic acid was found to interfere with the lactic acid determination, but a correction was made for this, $1 \mathrm{mg}$. pyruvic acid giving the same colour reaction as $0.06 \mathrm{mg}$. lactic acid. When large 
amounts of pyruvic acid were present in the sample a correction was made to the volatile acid; about $75 \%$ of the pyruvate distilled over in the first $200 \mathrm{ml}$. collected. Pyruvic acid was determined by the colorimetric method of Friedemann \& Haugen (1943). The method of Koepsell \& Sharpe (1951) for differentiating between pyruvic and $\alpha$-ketoglutaric acids was used on selected samples. No $\alpha$-ketoglutarate was found except in samples taken under anaerobic conditions at $\mathrm{pH} 7 \cdot 4$, where small amounts were detected. Ammonia was determined by the method of Conway (1957).

The opacity of the culture was not used as an estimate of the dry weight of organisms in it, as the relationship between dry weight and opacity may vary with the conditions of growth. However, the opacity reading provided a useful check as to whether the culture was in a steady state. The cell-carbon content was taken as $50.2 \%$ of the organism dry weight (Pirt, 1957).

Oxygen and carbon dioxide in the gas phase were determined with the Orsat apparatus as described by Pirt \& Callow (1958a). Analyses were always made on duplicate samples. There was an unavoidable error in the readings because no allowance could be made for the difference in water-vapour content between the gas fed to the culture and the effluent gas. However, as both were measured under the same conditions, the relative error from this source cannot have exceeded $3 \%$ and was probably less than $1.5 \%$. Towards the end of this work a paramagnetic oxygen analyser (Paramagnetic Oxygen Analyser Mark II, Servomex Controls Limited, Crowborough, Sussex) became available and was used in preference to the Orsat apparatus because of the greater speed and accuracy of the former; with it the oxygen content of the gas phase could be measured within $\pm 0.06 \%$. The partial pressure of oxygen in the gas phase over the culture was taken as equal to that in the effluent gas. The gas pressure in the vessel was less than $0.5 \mathrm{~mm}$. $\mathrm{Hg}$ above atmospheric pressure.

\section{RESULTS}

\section{The fate of glucose at different oxygen tensions}

Glucose in excess. To study the effect of dissolved oxygen tension on a culture with glucose in excess the culture was grown at a growth rate of $0.20 \mathrm{hr}^{-1}$ with nitrogen as the growth-limiting factor, and with excess glucose. The amount of glucose utilized was independent of that supplied provided that the supply was over $10 \mathrm{mg}$. $/ \mathrm{ml}$. The culture was at $\mathrm{pH} 6 \cdot 0$. The fate of the glucose carbon at different oxygen tensions is given in Fig. $1 a, b$. The culture showed three different states according to different ranges of dissolved oxygen tension. The 'excess oxygen state', which occurred at dissolved oxygen tensions above about $15 \mathrm{~mm}$. $\mathrm{Hg}$, had the following characteristics. The rate of oxygen uptake by the culture was constant and independent of the oxygen tension. The dissolved oxygen tension fell linearly with the oxygen partial pressure in the gas phase in accordance with equation 7 . About $95 \%$ of the carbon in the glucose utilized was accounted for in organisms, $\mathrm{CO}_{2}$ and pyruvic acid, with the remainder as 2:3 butanediol and ethanol. It appeared that the fermentation end-products (butanediol, ethanol) could not be completely eliminated by excess oxygen conditions with glucose in excess. The respiratory quotient (R.Q.) with excess oxygen and excess glucose was 0.8 . This low value is accounted for by the formation of pyruvate.

The second state, called the 'transition state' of the culture, was entered when the dissolved oxygen tension decreased below $15 \mathrm{~mm}$. $\mathrm{Hg}$; in this range the oxygen tension 
fluctuated between the values of $<1$ and $13 \mathrm{~mm}$. $\mathrm{Hg}$ (Fig. 2). Although the oxygen supply was maintained constant for over $48 \mathrm{hr}$ (about 13 generations) the fluctuations in the dissolved oxygen tension showed no sign of diminishing. With the medium used and specific growth rate $0.2 \mathrm{hr}^{-1}$ the fluctuations in dissolved oxygen tension were apparently permanent. Decrease of the gas partial pressure of oxygen still further eliminated the fluctuations in the dissolved oxygen tension and the dissolved oxygen reading became indistinguishable from the zero reading (i.e. $<1 \mathrm{~mm}$. $\mathrm{Hg}$ of $\mathrm{O}_{2}$ ). Thus the transition state was characterized by the occurrence of oscillations in the dissolved oxygen tension.

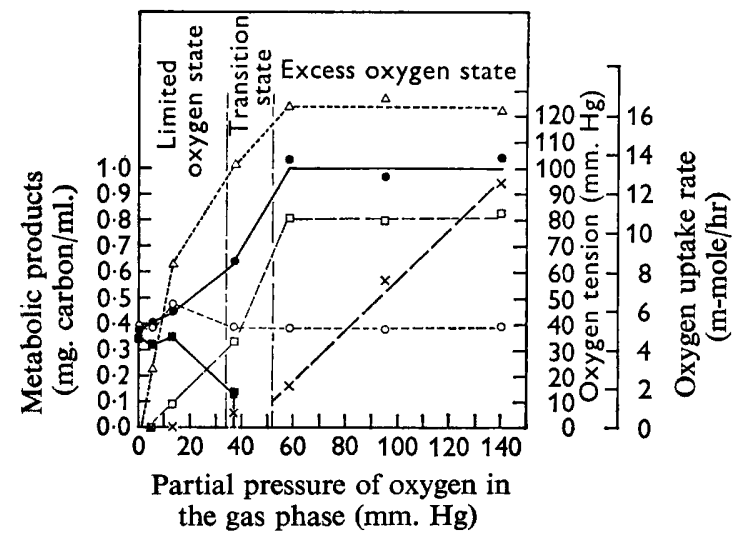

Fig. $1 a$

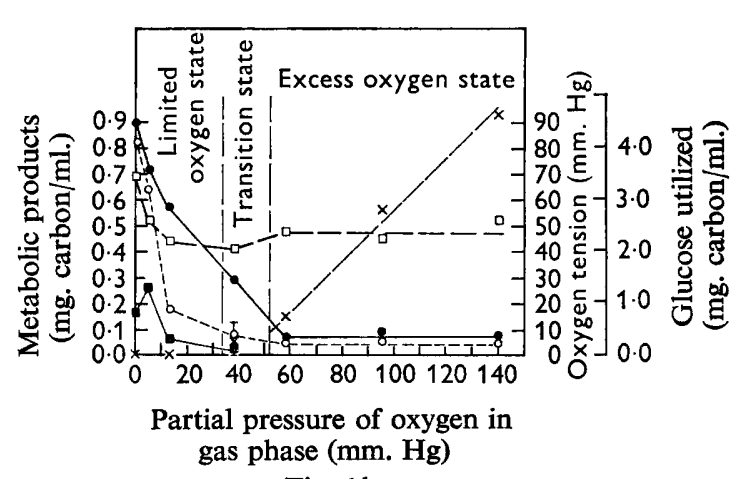

Fig. $1 b$

Fig. 1. $a, b$, Klebsiella aerogenes NCIB 8017. The fate of glucose at different oxygen tensions with nitrogen-limited growth and excess glucose, at $\mathrm{pH} 6 \cdot 0$. Dilution rate: $0 \cdot 20 \mathrm{hr}^{-1}$.

(a) $\times--\times$, dissolved oxygen tension; $\triangle--\triangle$, oxygen uptake rate. Products: $-\bullet$, organism dry weight; $\mathrm{O}--\mathrm{O}, \mathrm{CO}_{2} ; \square--\square$, pyruvic acid; $\square-\square$ volatile acid (as acetic). I, denotes the amplitude of oscillations in $\mathrm{O}_{2}$ tension.

(b) $\times--\times$, dissolved oxygen tension; $\square--\square$, glucose utilized. Products: $-0,2: 3$

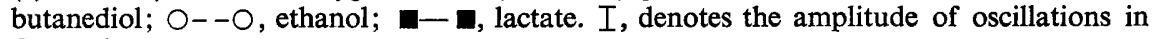
$\mathrm{O}_{2}$ tension.

When the oxygen supply was decreased below that which gave rise to the transition state, the third or 'limited oxygen state' was reached; here the dissolved oxygen was stable and indistinguishable from zero. Also in the limited oxygen state, the dry weight of organism and pyruvic acid production decreased with decrease in the oxygen supply, and the production of fermentation products butanediol, acetic acid, ethanol and lactate increased. It can be seen from Fig. $1 b$ that butanediol production started to increase at a higher oxygen supply rate than did ethanol production. Acetoin production was very low, accounting for only $0.01 \mathrm{mg}$. carbon $/ \mathrm{ml}$. The decrease in the dry weight organism at the lower oxygen tensions may be attributed to a decrease in stored polysaccharide rather than in cell protein since it was shown that growth was still nitrogen-limited and therefore probably the bacteria were still synthesizing the same amount of protein.

A similar pattern of results in response to changes in oxygen supply was obtained at growth rates of $0.10,0.40$ and $0.50 \mathrm{hr}^{-1}$, but at the higher growth rates of 0.40 and $0.50 \mathrm{hr}^{-1}$ the amounts of the products formed per ml. were much decreased. At a 
growth rate of $0 \cdot 10 \mathrm{hr}^{-1}$ no unsteady transition state was found. However, it may be that, at such a low growth rate, the unsteady state extends over a much narrower range of oxygen supplies and so was missed in these experiments. At growth rates of 0.40 and $0.50 \mathrm{hr}^{-1}$ the type of fluctuations found for the dissolved oxygen tension in the transition state (Fig. 3) differed from that found at $0.20 \mathrm{hr}^{-1}$. A more regular pattern was found at the higher growth rates and the amplitude of the oxygen tension oscillations was smaller.

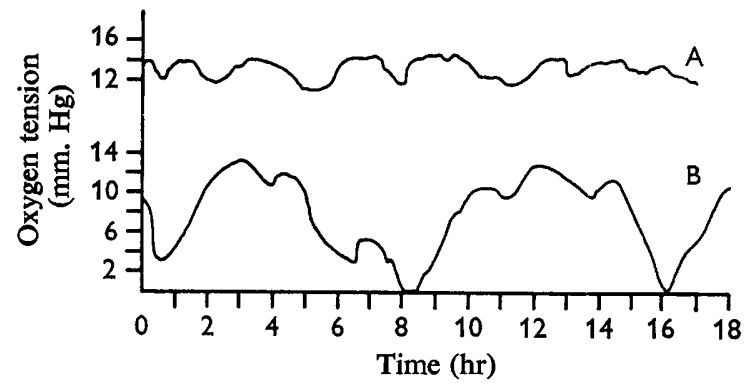

Fig. 2

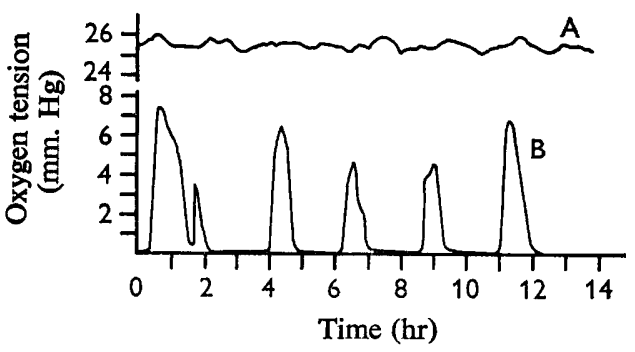

Fig. 3

Fig. 2. Klebsiella aerogenes NCIB 8017. Tracings of culture oxygen tension obtained with nitrogen-limited growth and excess of glucose at $\mathrm{pH} 6.0$. Dilution rate $0 \cdot 20 \mathrm{hr}^{-1}$. A, tracing obtained in the excess oxygen state. B, tracing obtained in the transition to the limited-oxygen state.

Fig. 3. Klebsiella aerogenes NCrB 8017. Tracings of culture oxygen tension obtained with nitrogen-limited growth and excess glucose at $\mathrm{pH} 6.0$; dilution rate, $0.40 \mathrm{hr}^{-1}$. A, tracing obtained in the excess oxygen state. B, tracing obtained in the transition to the limitedoxygen state.

Effect of an alkaline pH value. The metabolism of Klebsiella aerogenes may alter considerably with changes in $\mathrm{pH}$ value of the culture (Mickelson \& Werkman, 1938; Pirt \& Callow, 1958a), and so a culture was grown with excess glucose, controlled at

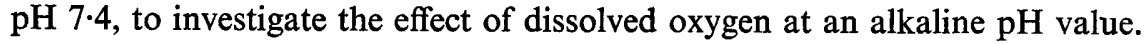

Again the metabolism fell into three states. In this case the transition state extended over a narrower range of dissolved oxygen tension. With a growth rate of $0.18 \mathrm{hr}^{-1}$ the dissolved oxygen tension remained below $1 \mathrm{~mm}$. $\mathrm{Hg}$, but once in every $24 \mathrm{hr}$ increased and fluctuated between 1.0 and $3.3 \mathrm{~mm}$. $\mathrm{Hg}$ about once per hour for $6 \mathrm{hr}$ and then decreased again to the steady value.

From Fig. 4 it can be seen that the pattern of metabolic end-products in the culture at $\mathrm{pH} 7.4$ differed considerably from that obtained at $\mathrm{pH} 6.0$. In the excess-oxygen state pyruvate production was considerably less than that obtained at $\mathrm{pH} 6.0$ and substantial amounts of acetic acid were formed. The transition from excess oxygen to limited oxygen was marked by a large increase in acetic acid production and a steep decrease in organism dry weight. The production of butanediol, ethanol, lactate and formate also increased immediately the oxygen supply became limiting. The organism dry weight reached a minimum before the culture was fully anaerobic, and pyruvate production did not cease completely under anaerobic conditions at $\mathrm{pH} 7.4$ as was the case at $\mathrm{pH}$ 6.0. Maximum butanediol and lactate production was reached, not under anaerobic conditions, but in the limited oxygen state. Acetoin was only detected in samples showing maximum amounts of diol, and then only accounted for $0.01 \mathrm{mg}$. 
carbon/ml. Very large amounts of volatile acids were formed at $\mathrm{pH} 7 \cdot 4$ under oxygenlimited conditions. The volatile acid was mostly acetic acid with the higher oxygen supply, but with decreased oxygen supply formic acid production increased until it accounted for about half as much glucose carbon as the acetic acid, i.e. the number of molecules of formic acid and acetic acid produced were equal.

Glucose-limited growth. To investigate how much the phenomena observed were a result of glucose being in excess, cultures were made with glucose as the growthlimiting substance, and with an excess of ammonium sulphate as $\mathrm{N}$ source. The glucose feed was adjusted to give a supply of about $2.5 \mathrm{mg} . / \mathrm{ml}$., the culture controlled at $\mathrm{pH}$ 6.0 and the growth-rate kept at $0 \cdot 16 \mathrm{hr}^{-1}$.

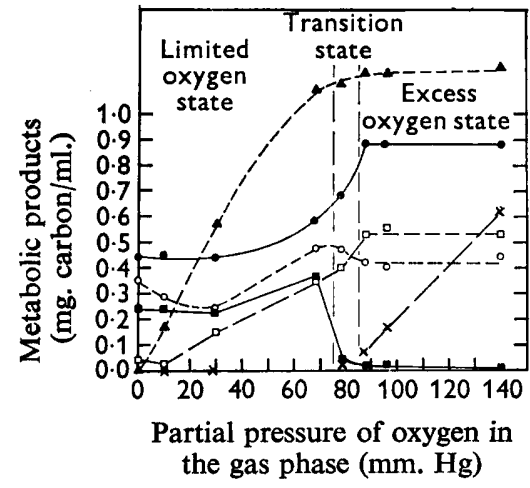

Fig. $4 a$

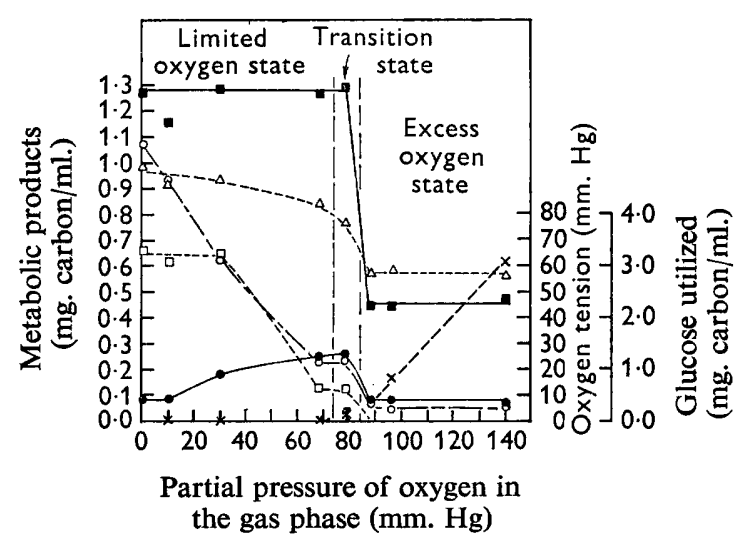

Fig. $4 b$

Fig. 4.a, b. Klebsiella aerogenes NCIB 8017. The fate of glucose at different oxygen tensions with nitrogen-limited growth and excess glucose at $\mathrm{pH} 7 \cdot 4$; dilution rate $0 \cdot 18 \mathrm{hr}^{-1}$.

(a) $\times---\times$, dissolved oxygen tension; $\boldsymbol{\Delta}---\boldsymbol{\Delta}$, oxygen uptake rate. Products: $-\mathbf{0}$ organism dry weight; $\mathrm{O}---\mathrm{O}, \mathrm{CO}_{2} ; \square---\square$, pyruvic acid.

(b) $\times---\times$, dissolved oxygen tension; $\triangle---\triangle$, glucose utilized. Products: $-\bullet, 2: 3$ butanediol; $O---O$, ethanol; $\square-\square$, acetic acid; $\square---\square$, formic acid. I, denotes the amplitude of oscillations in $\mathrm{O}_{2}$ tension.

The influence of oxygen tension on the fate of glucose is shown in Fig. 5. Again the excess oxygen, the transition and the limited oxygen states were apparent. In the excess oxygen state $95-100 \%$ of the glucose carbon was recovered as organisms and $\mathrm{CO}_{2}$, and pyruvic acid did not accumulate. In keeping with the complete oxidation of glucose, the R.Q. was 1.02 . In the limited oxygen state the organism dry weight and the $\mathrm{CO}_{2}$ production decreased with the decreased oxygen supply and the glucose-carbon appeared instead in butanediol, ethanol and volatile acid. Acetoin was only detected when no oxygen was supplied to the culture; it then accounted for $0.07 \mathrm{mg}$. carbon $/ \mathrm{ml}$.

The transition state was characterized by continuous oscillations in the dissolved oxygen tension. As with excess glucose the oscillations occurred at oxygen tensions below about $15 \mathrm{~mm}$. $\mathrm{Hg}$ (Fig. 6). The dropwise addition of glucose solution caused the oxygen tension to oscillate over a range of about $1 \mathrm{~mm}$. $\mathrm{Hg}$. These small oscillations were imposed on the major oscillations. Since the respiration rate was glucoselimited it increases and decreases slightly in rhythm with the drops of glucose solution. The pattern of oscillations in oxygen tension in the transition state with glucoselimited growth was much more irregular than was the case with excess glucose. 
In Fig. 5 it is apparent that the respiration rate $\left(q_{\mathrm{O}_{2}}\right.$, expressed as m-mole $\mathrm{O}_{2}$ consumed/g. organism dry wt/hr) increased by about $33 \%$ in the limited oxygen state. This may be taken to represent a true increase in the oxygen uptake rate per bacterium because, with glucose-limited growth, polysaccharide is not stored in the organism (Holme, 1957) and so the increase apparent in $q_{\mathrm{O}_{2}}$ cannot be explained by a decrease in the mass of individual bacteria.

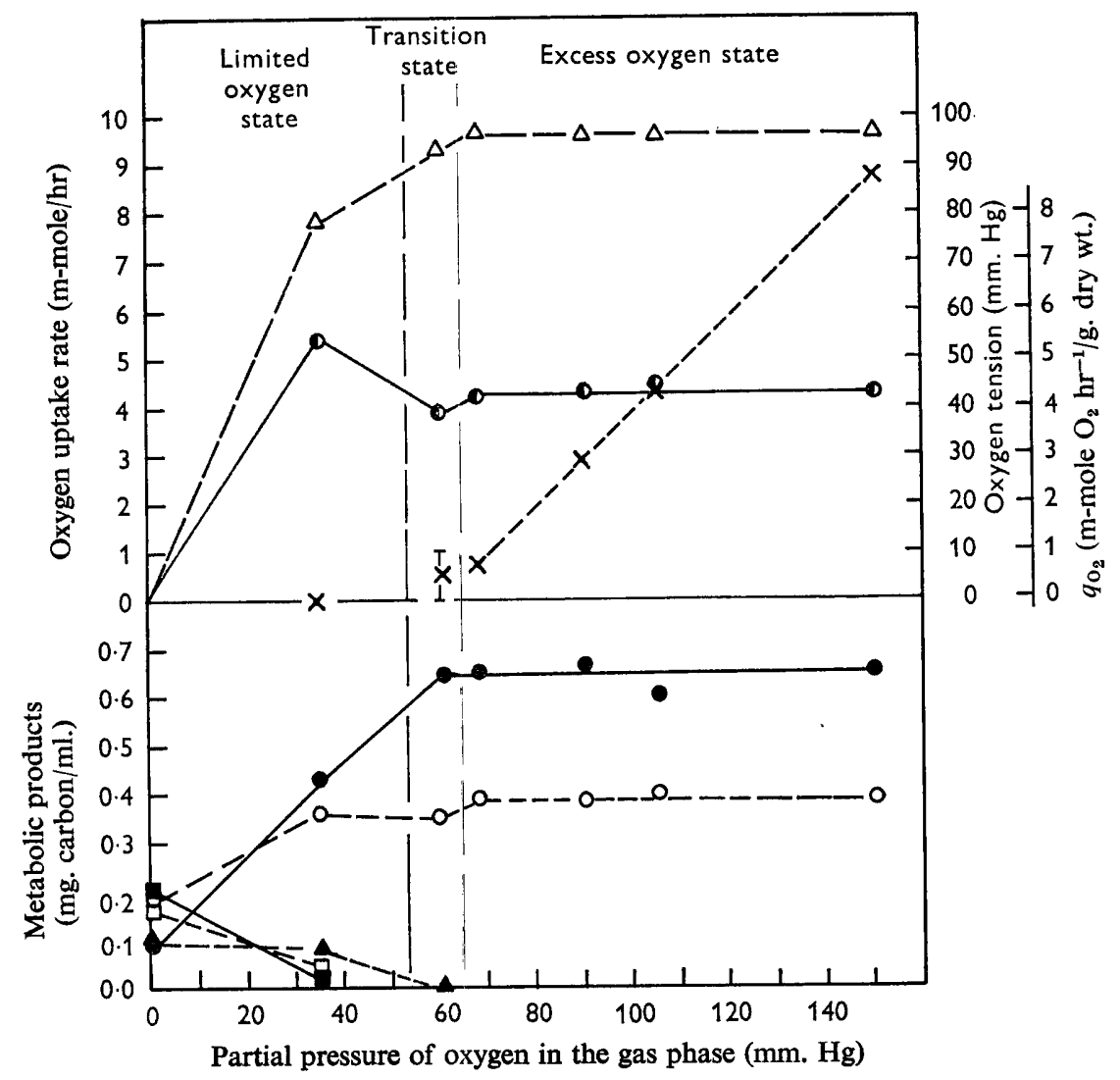

Fig. 5. Klebsiella aerogenes NCIB 8017. The fate of glucose at different oxygen tensions with glucose-limited growth at $\mathrm{pH} 6 \cdot 0$, dilution rate $0.16 \mathrm{hr}^{-1}$. Glucose concentration in entering medium $2.6 \mathrm{mg} . / \mathrm{ml} . \times---x$, dissolved oxygen tension: $\triangle---\Delta$, oxygen uptake rate; $-\mathrm{O}$, respiration rate $\left(q_{\mathrm{O}_{2}}\right)$. Products: $\bullet-\bullet$, organism dry weight; $\mathrm{O}---\mathrm{O}, \mathrm{CO}_{2}$; - - $2: 3$ butanediol; $\square--\square$, ethanol; $\Delta--\Delta$, volatile acid. $I$, denotes the amplitude of oscillations in $\mathrm{O}_{2}$ tension. Glucose utilization was over $97 \%$ of that supplied, under all conditions.

\section{The transition state}

The possibility that the fluctuations in oxygen tension obtained during the transition phase were not caused by the growing organisms but were an artifact due, for example, to small variations in oxygen supply, was investigated as follows. The aeration conditions were simulated by the oxidation of a sulphite solution (Cooper, Fermstrom \& Miller, 1944) in the culture vessel. The sulphite solution $(0.17 \mathrm{M})$ containing $\mathrm{CuSO}_{4}$ $(0.0005 \mathrm{M})$ was fed into the culture vessel at a constant rate to give a constant oxygen uptake rate in the solution. The sulphite feed-rate was adjusted to decrease the dis- 
solved oxygen tension to about $5 \mathrm{~mm} . \mathrm{Hg}$, that is, well within the transition state range of a culture. With the same stirring rate, gas flow rate and gas phase partial pressure of oxygen which gave oscillations in the culture it was not found possible to induce oscillations in the dissolved oxygen tension in the sulphite solution. The oxygen tension in the sulphite solution varied less than $\pm 0.75 \mathrm{~mm}$. Hg. Since the oscillations in the dissolved oxygen tension in the transition state of a culture were up to ten times as great, they cannot be attributed to variation in the oxygen supply rate.

The meaning of changes in dissolved oxygen tension during the transition state. Although the patterns of fluctuations obtained during the transition phase varied with different conditions of growth, there were certain basic similarities between the patterns. The lowest dissolved oxygen tension reached in each case was close to zero. This was followed by a steep increase in the oxygen tension reading, which then remained high for a period before decreasing again. However, the maximum oxygen tension reached during the fluctuations, and the frequency and length of the peaks and troughs, varied considerably. From the similarities, and the fact that the fluctuations were only obtained during the transition from aerobic to anaerobic metabolism,

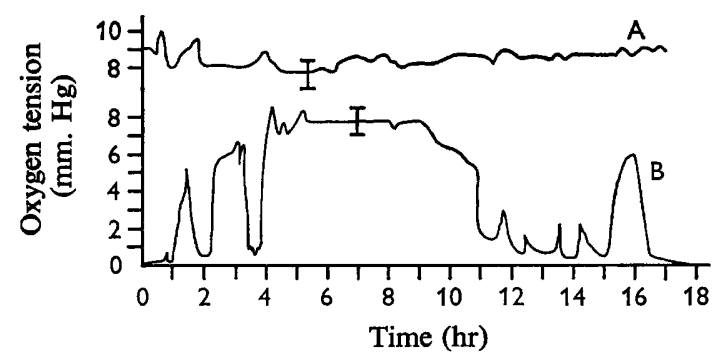

Fig. 6

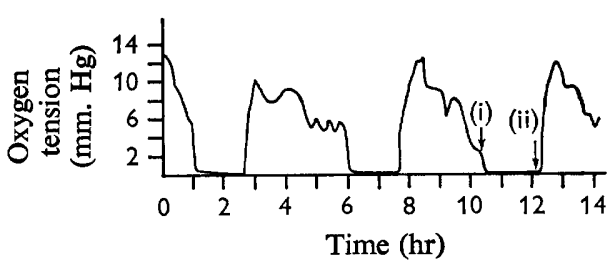

Fig. 7

Fig. 6. Klebsiella aerogenes NCIB 8017. Tracings of culture oxygen-tension reading, obtained with glucose-limited growth at $\mathrm{pH} 6.0$; dilution-rate $0.16 \mathrm{hr}^{-1}$. A, tracing obtained in the excess oxygen state. B, tracing obtained in the transition to the limited oxygen state. I, indicates the amplitude of oscillations (of frequency $45 / \mathrm{hr}$ ) superimposed on these tracings, which were caused by the drop-wise addition of glucose.

Fig. 7. Klebsiella aerogenes NCIB 8017. Tracing of culture oxygen-tension readings obtained with nitrogen-limited growth and glucose in excess at $\mathrm{pH} 6.0$, dilution-rate, $0 \cdot 20 \mathrm{hr}^{-1}$. Points (i) and (ii) are referred to in Table 2.

it would seem that, in spite of the differences in the actual form of the fluctuations, the states of flux obtained were all homologous.

The phenomenon of an unsteady state between aerobic and anaerobic metabolism was further investigated in the following way. A transition state was obtained in a culture with a growth rate of $0.20 \mathrm{hr}^{-1}$ at $\mathrm{pH} 6.0$ with glucose in excess. The oxygen tension in the culture was followed by using a Mackereth electrode. Figure 7 shows the tracing obtained. Another Mackereth electrode was used to follow the changes in oxygen partial pressure in the effluent gas. This electrode was inserted into a rubber tube ( $25 \mathrm{~mm}$. diameter) joined to the effluent gas pipe-line from the culture, so that the effluent gas flowed out around the membrane. There was no temperature-compensating device with this system, but it was found, by using a temperature recorder, that there were no sudden changes of room temperature and a steady recording could be obtained. 
The oxygen uptake rate in this experiment was measured by means of the paramagnetic oxygen analyser.

It was thus found that every increase in oxygen tension recorded in the culture corresponded to an increase in oxygen partial pressure in the effluent gas, and every sudden decrease in oxygen tension recorded in the culture corresponded to a sudden decrease in oxygen partial pressure in the effluent gas. An increase in oxygen partial pressure in the effluent gas accompanied by an increase in the dissolved oxygen tension must reflect a decrease in the oxygen uptake rate of the culture. A decrease in oxygen partial pressure in the gas phase accompanied by a decrease in the dissolved oxygen tension must be the result of an increase in the oxygen uptake rate of the culture.

Table 1. Klebsiella aerogenes NCIB 8017. Comparison between oxygen uptake rates in different aerobic states

Growth rate $0.20 \mathrm{hr}^{-1}, \mathrm{pH} 6.0$, glucose in excess.

$\begin{array}{ccccc}\begin{array}{c}\text { Culture } \\ \text { state }\end{array} & \begin{array}{c}\text { Oxygen } \\ \text { in gas } \\ \text { supply } \\ (\%)\end{array} & \begin{array}{c}\text { Dissolved } \\ \text { oxygen } \\ \text { tension } \\ (\mathrm{mm} . \mathrm{Hg})\end{array} & \begin{array}{c}\text { Oxygen } \\ \text { uptake } \\ \text { rate } \\ (\mathrm{m}-\mathrm{mole} / \mathrm{hr})\end{array} & \begin{array}{c}\text { Number } \\ \text { of } \\ \text { samples }\end{array} \\ \begin{array}{c}\text { Excess oxygen } \\ \text { Transition (at high oxygen } \\ \text { tension in cycle) }\end{array} & 17 \cdot 0-20 \cdot 9 & 27 \cdot 0-70 \cdot 0 & 16 \cdot 5 \pm 0.34 & 5 \\ \begin{array}{c}\text { Transition (at low oxygen } \\ \text { tension in cycle) }\end{array} & 16 \cdot 2 & 11 \cdot 0-13 \cdot 0 & 15 \cdot 15 \pm 0 \cdot 26 & 2 \\ \end{array}$

Table 2. Klebsiella aerogenes NCIB 8017. Comparison between the amounts of growth and $\mathrm{CO}_{2}$ production in the excess oxygen state and in the transition to the limited oxygen state

Excess glucose present, $\mathrm{pH} 6 \cdot 0$, dilution rate $0 \cdot 2 \mathrm{hr}^{-1}$.

Culture state
Excess oxygen
Transition at high dissolved oxygen
tension (about $5 \mathrm{~mm}$. $\mathrm{Hg}$, point $i$ in
Fig. 7)
Transition at low dissolved oxygen
tension ( $<0.2 \mathrm{~mm}$. Hg; point ii in
Fig. 7)

Excess oxygen

Transition at high dissolved oxygen tension (about $5 \mathrm{~mm}$. $\mathrm{Hg}$, point $i$ in

Fig. 7)

$\begin{array}{cc}\begin{array}{c}\text { Organism dry } \\ \text { weight carbon } \\ (\mathrm{mg} / \mathrm{ml} .)\end{array} & \begin{array}{c}\mathrm{CO}_{2} \text {-carbon } \\ (\mathrm{mg} . / \mathrm{ml} .)\end{array} \\ 1.09 \pm 0.06 & 0.39 \pm 0.02 \\ 1.15 & 0.37 \\ 1.11 & 0.39 \\ 0.89 & 0.50 \\ 0.83 & 0.54\end{array}$

Table 1 shows the results of measurements of oxygen uptake rates taken at various points in the transition state. It can be seen that the oxygen uptake rate was significantly higher when the oxygen tension had decreased to the minimum, than when the oxygen tension was at the maximum of the cycle. Also it can be seen from Table 1, that when the culture was in the transition state and the oxygen tension was at the minimum, the oxygen uptake rate was significantly higher than that obtained with excess oxygen. Since the organism dry weight fell about $20 \%$ in the transition state (Table 2) and the amount of nitrogen taken up by the organisms was the same or slightly less, the increased oxygen uptake cannot be attributed to increase in cell mass or cell nitrogen. 
Further evidence of an increase in the respiration rate when the oxygen tension of the culture decreased to the minimum in the transition state is shown in Fig. 8. With dissolved oxygen tensions above about $12 \mathrm{~mm}$. $\mathrm{Hg}$ the relation between the oxygen tensions in the liquid and the gas phases was linear, in accordance with equation 7. However, some of the values of the dissolved oxygen tension during the transition phase lay below the extrapolated straight line. Since the aeration conditions were constant ( $K$ in equation 7 was constant) the increased slope of the line must indicate an increase in the oxygen uptake rate $(N)$.

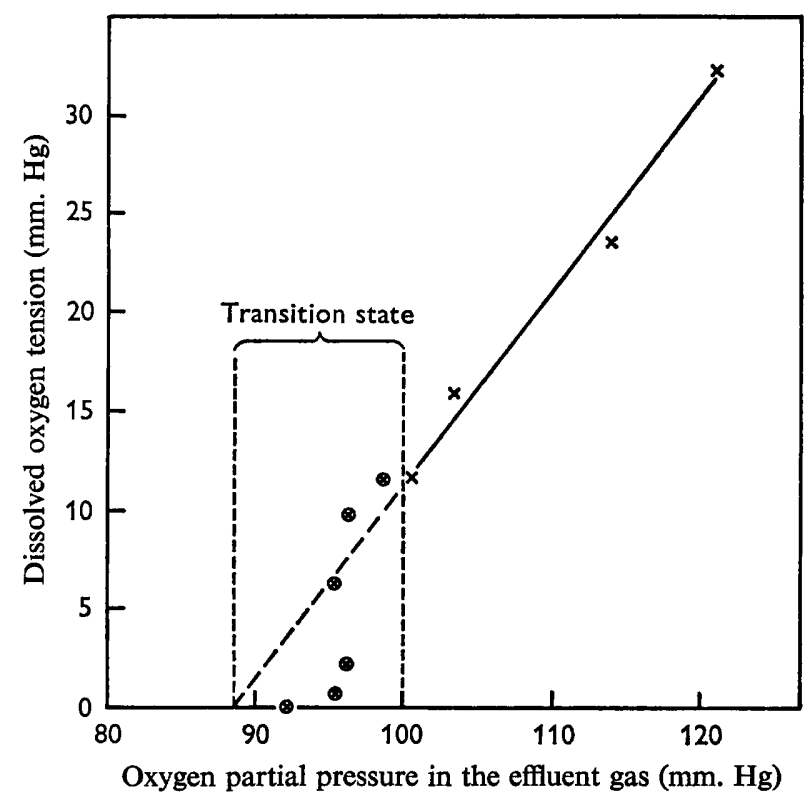

Fig. 8. Dissolved oxygen tension as a function of the partial pressure of oxygen in the gas phase. $x$, oxygen tension readings during the excess oxygen state. $\otimes$, oxygen readings during the transition to the limited oxygen state.

The fate of glucose in the transition phase when the oxygen tension was high and when at the minimum (points $i$ and ii in Fig. 7) was determined and compared with that in the excess oxygen state. The pyruvate production was the same, and there were slight increases in the amounts of butanediol, ethanol and volatile acid in the transition state. The only marked differences were in organism dry weight and $\mathrm{CO}_{2}$ production; these are shown in Table 2. In the transition state when the oxygen tension was minimal, the organism dry weight decreased and $\mathrm{CO}_{2}$ production increased greatly, although the glucose utilization did not change. Thus in the transition phase increased respiration rate was accompanied by an increased $\mathrm{CO}_{2}$ production rate and a decrease in the yield of organism/g. glucose utilized.

Considering the large change in $\mathrm{CO}_{2}$ production during the transition phase, it seemed possible that some of the changes occurring might be caused by changes in $\mathrm{CO}_{2}$ partial pressure. To test this the partial pressure of $\mathrm{CO}_{2}$ in the gas phase was increased by feeding $5 \% \mathrm{CO}_{2}$ into the gas supply and adjusting the nitrogen flow to keep the oxygen content the same. The increase in $\mathrm{CO}_{2}$ partial pressure (about threefold) had no detectable effect on the fluctuations in oxygen tension. 
Response to induced changes in oxygen tension in the transition state. Experiments were made to find whether the changes in oxygen uptake rate in the transition phase were a direct response to changes in the dissolved oxygen tension. Changes in dissolved oxygen tension were brought about by altering the percentage of oxygen in the gas supply until the desired oxygen tension was reached, when the oxygen supply was returned to its original value. The experiments were repeated with alteration of the oxygen tension by changing the rate of flow of gas through the culture, to check that the organisms were not responding to nitrogen partial pressure. The results were the same in each case.

When the dissolved oxygen tension reading was in the 'high' part of the cycle (i.e. the oxygen uptake rate was low) it was found that decreasing the dissolved oxygen tension to $6 \mathrm{~mm}$. $\mathrm{Hg}$ had no effect on the oxygen uptake rate. When the oxygen feed was returned to its previous value, the dissolved oxygen tension increased again. However, when the dissolved oxygen tension was decreased to $4 \mathrm{~mm}$. $\mathrm{Hg}$, it did not increase again on returning the oxygen supply to its previous value, but continued to decrease until it reached the zero reading. The oxygen uptake rate had increased. The oxygen tension then remained low for about $1.5 \mathrm{hr}$ before showing a sudden spontaneous increase to about $13 \mathrm{~mm}$. Hg, like that found in the cycle of fluctuations shown in Fig. 7. This response was obtained at any point on the 'high' part of the cycle of oxygen tension fluctuations, whether the oxygen tension had just increased from zero or whether it had remained high for more than $2 \mathrm{hr}$. It was noticed, however, that the longer the culture was maintained in the fluctuating state the lower was the threshold oxygen tension at which the respiration rate was stimulated. Initially the threshold was about $4.0 \mathrm{~mm}$. $\mathrm{Hg}$ of $\mathrm{O}_{2}$, but after $24 \mathrm{hr}$ it was about $2.0 \mathrm{~mm}$. $\mathrm{Hg}$. The same trend can be seen in Fig. 7, where in the first fluctuation, the sudden decrease in oxygen tension occurred when the reading had reached about $4.0 \mathrm{~mm}$. $\mathrm{Hg}$, but in the third cycle the threshold was closer to $2 \cdot 0 \mathrm{~mm}$. $\mathrm{Hg}$.

When the dissolved oxygen tension was in the 'low' part of the cycle of fluctuations, that is, the oxygen uptake rate was stimulated, increasing the dissolved oxygen tension to $5 \mathrm{~mm}$. $\mathrm{Hg}$ had no effect on the oxygen uptake rate. When the oxygen supply was returned to its previous value the oxygen tension decreased again. When the dissolved oxygen tension was increased to $10 \mathrm{~mm}$. $\mathrm{Hg}$, however, the oxygen tension did not decrease again on returning the oxygen supply to its previous value, but continued to increase; it increased to $17 \mathrm{~mm}$. $\mathrm{Hg}$ and remained at this value, that is, the culture entered the 'high' part of the cycle. Hence the respiration rate was partially inhibited by an increase in the dissolved oxygen tension to $10 \mathrm{~mm}$. $\mathrm{Hg}$.

The spontaneous increase in oxygen tension during the 'low' part of the cycle might be attributed to a continued decrease in the actual oxygen tension which the electrode was not sensitive enough to show; then, when the oxygen tension reached a certain value below the sensitivity of the instrument (about $0.2 \mathrm{~mm} . \mathrm{Hg}$ ) the organism responded by decreasing the respiration rate. To test this possibility when the culture was in the 'low' part of the cycle (i.e. while the respiration was in the stimulated state and the oxygen tension reading was minimal) the oxygen supply to the culture was decreased temporarily. This was done by stopping the air supply to the culture and feeding pure nitrogen for a few minutes. The oxygen supply was then returned to its previous value. There was no apparent decrease in the oxygen demand of the culture; the oxygen tension remained low after the partial pressure of oxygen in the effluent 
gas was restored to its previous value. This procedure was repeated at various points on the 'low' part of the cycle, always with no response from the culture. Therefore, it was concluded that the inhibition of oxygen uptake rate which occurred spontaneously in the cycle was not an immediate response to a decrease in dissolved oxygen tension to a very low critical value, below the sensitivity of the electrode.

Interpretation of the fluctuations in oxygen tension. From the observations in the above experiments, the fluctuations shown in Fig. 7 can be interpreted as follows. When the dissolved oxygen tension decreased below a certain threshold value (about $5 \mathrm{~mm} . \mathrm{Hg}$ ) the culture responded by increasing the respiration rate so that there was a sudden fall in dissolved oxygen tension. The increased oxygen uptake rate was maintained for about $1.5 \mathrm{hr}$ and was accompanied by a decrease in organism production and an increase in $\mathrm{CO}_{2}$ production. When the dissolved oxygen tension had been at the minimum for about $1.5 \mathrm{hr}$ there was a sudden reversion to the lower respiration rate, causing a rapid increase in dissolved oxygen tension. It is postulated that this reversion to the lower respiration rate was caused by the build-up of an inhibitor. The inhibitor quickly disappeared once the oxygen tension increased, because under these conditions decreasing the dissolved oxygen tension below the threshold value of about $5 \mathrm{~mm}$. $\mathrm{Hg}$ caused the culture to take up oxygen at the higher rate again. When the dissolved oxygen tension increased to about $13 \mathrm{~mm} . \mathrm{Hg}$ the oxygen uptake rate was at first a little lower than that obtained under fully aerobic conditions, because the organism dry weight decreased while the oxygen tension was low. The slow decrease in dissolved oxygen tension which followed was probably caused by the increase in organism dry weight so that the oxygen uptake rate increased slowly.

Although this interpretation was deduced from a regular pattern of fluctuations such as was obtained in Fig. 7 the same principles can account for the more irregular types of fluctuations obtained in the transition phase. The fact that the pattern of the fluctuations varied from one culture to another, even when the conditions of growth were apparently similar (as in Fig. 2 and 7), suggests that the past history of the culture has an influence on its behaviour in the transition phase. In the case illustrated in Fig. 7, the culture had not been kept growing in the excess oxygen phase as long as in the experiment depicted in Fig. 2.

\section{DISCUSSION}

In response to changes in dissolved oxygen tension the continuous culture of Klebsiella aerogenes NCIB 8017 exhibited three states. In the excess oxygen state obtained at oxygen tensions above $10-15 \mathrm{~mm}$. $\mathrm{Hg}$ the end-products of glucose metabolism and the respiration rate were independent of dissolved oxygen tension. In the transition state the oxygen uptake rate of the culture varied although the aeration conditions were constant. The varying oxygen uptake rate caused the dissolved oxygen tension to oscillate within the range $<0 \cdot 2-15 \mathrm{~mm}$. $\mathrm{Hg}$, the range of the fluctuations seeming to depend upon the conditions and the history of the culture. In the limited-oxygen state the dissolved oxygen tension decreased to $<1 \mathrm{~mm}$. $\mathrm{Hg}$ and the oxygen uptake rate of the culture was dependent on the oxygen supply rate. Pirt (1957) characterized three phases in the metabolism of Aerobacter cloacae: a fully aerobic, a partially aerobic and an anaerobic phase. The present observation of an unsteady state in the transition between a fully aerobic and a partially aerobic metabolism is new. 
In the excess oxygen state when the growth of Klebsiella aerogenes was glucoselimited, all the glucose carbon could be accounted for as organisms and carbon dioxide, and the R.Q. was 1. However, when growth was nitrogen-limited and glucose was supplied in excess, a large part of the glucose utilized was converted into pyruvate and into material (probably glycogen) stored in the organisms; in this state the R.Q. was $\mathbf{0 . 8}$. It is interesting to note that small amounts of fermentation products (butanediol, ethanol) were found in the excess oxygen state when glucose was in excess, although, since the amounts were small, it might be argued that in spite of the immediate cooling of samples the products could have been formed after the sample had been removed from the culture, when the oxygen supply was cut off. In the limited oxygen state, fermentative pathways began replacing the aerobic oxidation of glucose as the major energy-generating mechanism.

The production of formic and acetic acids in equimolar amounts under anaerobic conditions at $\mathrm{pH} 7 \cdot 4$ would seem to confirm suggestions by other workers (Wood, 1961; Hadjipetrou, Gerrits, Teulings \& Stouthamer, 1964) that the thioclastic cleavage of pyruvate occurs in Aerobacter. The breakdown of formic acid to $\mathrm{H}_{2}$ and $\mathrm{CO}_{2}$ is suppressed at an alkaline $\mathrm{pH}$ as reported by Blackwood, Neish \& Ledingham (1956). Wood (1961) suggested that the ethanol formed by Aerobacter is produced from the acetyl phosphate or acetyl CoA which is produced by the cleavage of pyruvate. If this were so, then the number of molecules of formic acid produced at an alkaline $\mathrm{pH}$ by Klebsiella aerogenes should equal the number of molecules of acetic acid + ethanol formed. It can be seen from Fig $4 b$ that a large amount of ethanol was formed under anaerobic conditions at $\mathrm{pH} \mathbf{7 \cdot 4}$, although formic and acetic acids were formed in equimolar amounts. Thus it would seem that ethanol was not produced via the thioclastic cleavage of pyruvate under these conditions.

The experiments reported here are concerned with the change from growth in presence of excess oxygen to growth with limited oxygen. The reverse change, from steady-state anaerobic to aerobic growth, was not studied because it was found that when the culture was kept anaerobic for about 10 or more generations the change to aerobic growth took several days ( 20 or more generations) to reach a steady state; this suggests that complex adaptive changes or selection of variants were involved.

The concept of 'critical oxygen tension' as the tension below which the oxygen uptake rate of an organism decreases with a decrease in oxygen tension, has been widely quoted by workers since its introduction by Gerard \& Falk (1931). However, from the work of Moss (1956) and White (1963) there are indications that growing organisms may not respond in such a straightforward manner at low oxygen tensions. This has been confirmed in the present work, where it has been shown that the response of Klebsiella aerogenes to decreased oxygen tension may involve an increase in respiration rate rather than a decrease. A modified definition of 'critical' oxygen tension to meet the new situation would be: the oxygen tension above which the respiration rate of an organism is independent of changes in dissolved oxygen tension; below this tension the oxygen uptake rate of the organism may increase or decrease in response to a decrease in oxygen tension, according to cultural conditions.

From the interpretation of the fluctuations in oxygen tension obtained in the transition phase, it would seem that a growing culture of Klebsiella aerogenes may show two types of aerobic respiration: (i) at oxygen tensions above the 'critical' value the oxygen uptake rate of $K$. aerogenes is constant and independent of oxygen tension; (ii) when the 
oxygen tension is decreased below the 'critical' value the organism at first takes up oxygen at a higher rate and converts more glucose to $\mathrm{CO}_{2}$ and less to organism.

The actual value of the critical oxygen tension in the cultures investigated here varied with cultural conditions from 2 to about $10 \mathrm{~mm}$. $\mathrm{Hg}$. Induction of respiratory enzymes at low oxygen tensions may be involved. However, in the case studied here the response to a decrease in oxygen tension below the 'critical' value was so very rapid (less than $5 \mathrm{~min}$.) that it seems doubtful whether the simple induction of enzymes could explain this. Similarly, when the oxygen tension was increased above the 'critical' value the culture immediately reverted to the lower oxygen uptake rate.

It may be thought that there was some connexion between the oscillations in respiration rate and the generation time of the organism possibly involving synchronization of cell division. This would be ruled out, firstly because, even when the oscillations in oxygen tension were fairly regular the period of the oscillations was always much greater than the generation time of the organisms; and secondly because the rise and fall in the respiration rate occurring with dissolved oxygen concentration approximately in the range $0-10 \mathrm{~mm}$. Hg could be produced at will, irrespective of the previous history of the culture.

Many workers have shown that it is the rate of transfer of electrons to the terminal oxidase which limits the oxygen uptake rate under fully aerobic conditions (Winzler, 1941 ; Chance, 1952; Longmuir, 1957), so that changes in the terminal oxidase system alone would not be expected to increase the respiration rate. If the electron transport chain were the rate-limiting factor for fully aerobic respiration, then an increase in respiration rate at low oxygen tensions might be brought about by the functioning of an alternative pathway for electron transport.

In recent years there have been reports indicating that ADP and ATP concentrations are important in the control of glucose utilization (Barker, Khan \& Solomos, 1964; Maitra, Estabrooke \& Chance, 1963) and of electron transport systems (Chance, 1961). It is tempting to propose that the stimulation of respiration in Klebsiella aerogenes by a low oxygen tension is caused by a decreased ATP yield which accompanies either a change to an alternative electron transport pathway or the uncoupling of oxidative phosphorylation. Uncoupling of phosphorylation similarly can cause more rapid electron transfer and oxygen uptake (Racker, 1965).

We gratefully acknowledge the technical assistance of Mr. F. E. Bush, a research grant in aid of this work from the Science Research Council, a Science Research Council studentship to Dr D. E. F. Harrison, and the loan of equipment from the British Oxygen Company.

\section{REFERENCES}

Barker, J., Khan, M. A. A. \& Solomos, T. (1964). Mechanism of the Pasteur effect. Nature, Lond. 201, 1126.

BAUmberger, J. P. (1939). The relation between 'oxidation-reduction potential' and the oxygen consumption rate of yeast cell suspensions. Cold Spr. Harb. Symp. quant. Biol. 7, 195.

Bishop, J. M. (1959). Measurement of blood oxygen tension. Proc. R. Soc. Med. $53,177$.

Blackwood, A. C., Neish, A. C. \& Ledingham, G. A. (1956). Dissimilation of glucose at controlled $\mathrm{pH}$ values by pigmented and non-pigmented strains of Escherichia coli. J. Bact. $72,497$.

Bracken, A., Glover, J. H. \& Longmuir, I. S. (1960). A new method of oxygen determination. Lancet, ii, 411.

Callow, D. S. \& PIRT, S. J. (1956). Automatic control of $\mathrm{pH}$ value in cultures of micro-organisms. J. gen. Microbiol. 14, 661. 
Chain, E. B. \& Gualand, G. (1954). Aeration studies. R.C. Ist. sup. Sanità. (English ed.) 17, 5.

CHANCE, B. (1952). Spectra and reaction kinetics of respiratory pigments of homogenised and intact cells. Nature, Lond. 169, 215.

Chance, B. (1957). Cellular oxygen requirements. Fedn. Proc. Fedn. Am. Socs exp. Biol. $16,671$.

CHANCE, B. (1961). Reversal of electron transfer in the respiratory chain. In Biological Structure and Function, vol. 2. New York; Academic Press.

CHIN, C. H. (1950). Effect of aeration on the cytochrome systems of the resting cells of brewers' yeast. Nature, Lond. 165, 926.

Conway, E. J. (1957). Micro-diffusion Analysis and Volumetric Error., 4th ed. New York: Lockwood.

Cooper, C. M., Fernstrom, G. A. \& Miller, S. A. (1944). Performance of agitated gas-liquid contractors. Ind. Engng. Chem. 36, 504.

Ephrussi, B., Slonimski, P. P. \& Perrodin, G. (1950). La synthèse adaptive des cytochromes chez la levure de boulangerie. Biochim. biophys. Acta 6, 256.

FINN, R. K. (1954). Agitation-aeration in the laboratory and in industry. Bact. Rev. 18, 254.

Friedeman, T. E. \& Haugen, G. E. (1943). Pyruvic acid II. The determination of keto acids in blood and urine. J. biol. Chem. 147, 415.

GERARD, R. W. \& FALK, I. S. (1931). Observations on the metabolism of Sarcina lutea. I. Biol. Bull. mar. biol. Lab. 60, 213.

GraY, C. T., WIMPENNY, J. W. T. \& Mossman, M. R. (1966). Regulation of metabolism in facultative bacteria. II. Effect of aerobiosis, anaerobiosis and nutrition on the formation of Krebs enzymes in Escherichia coli. Biochem. biophys. Acta 117, 33.

Haditetrou, L. P., Gerrits, J. K., Teulings, F. A. G. \& Stouthamer, A. H. (1964). Relation between energy production and growth of Aerobacter aerogenes. J. gen. Microbiol. $36,139$.

HeRBert, D. (1958). Some principles of continuous culture. In Recent Progress in Microbiology. Symposia VII Int. Congr. Microbiol. p. 381. Stockholm: Almquist \& Wiksell.

HLXON, A. W. \& GADEN, E. L. (1950). Oxygen transfer in submerged fermentations. Ind. Engng Chem. 42, 1792.

Holme, T. (1957). Continuous culture studies on glycogen synthesis in Escherichia coli в. Acta chem. scand. 11, 763.

KINSEY, D. W. \& BotTomLey, R. A. (1963). Improved electrode system for determination of oxygen tension in industrial applications. J. Inst. Brew. 69, 164.

KoEPSELl, H. J. \& SHARPE, E. S. (1951). Microdetermination of pyruvic and $\alpha$-ketoglutaric acids. Arch. Biochem. Biophys. 38, 443.

LONGMUIR, I. S. (1954). Respiration rate of bacteria as a function of oxygen concentration. Biochem.J. 57, 81.

LoNGMUIR, I. S. (1957). Respiration rate of rat-liver cells at low oxygen concentrations. Biochem. J. $65,378$.

MACKERETH, F. J. H. (1964). An improved galvanic cell for determination of oxygen concentration in fluids. $J$. scient. Instrum. 41, 38.

Maclennan, D. G. \& PIRT, S. J. (1966). Automatic control of dissolved oxygen tension in stirred microbial cultures. J. gen. Microbiol. 45, 289.

Maitra, P. K., Estabrook, R. W. \& Chance, B. (1963). Yeast as a model for the study of the regulation of cellular metabolism. p. 491, C.N.R.S. International Colloquium, Marseille.

Mickelson, M. \& WeRkMAN, C. H. (1938). Influence of $\mathrm{pH}$ on the dissimilation of glucose by Aerobacter indologenes. J. Bact. 36, 67.

Moss, F. (1952). The influence of oxygen tension on respiration and cytochrome $a_{2}$ formation of Escherichia coli. Aust. J. exp. Biol. med. Sci. 30, 531.

Moss, F. (1956). Adaptation of the cytochromes of Aerobacter aerogenes in response to environmental oxygen tension. Aust. J. exp. Biol. med. Sci. 34, 395.

Phillips, D. H. \& Johnson, M. J. (1961). Aeration and fermentation. J. biochem. microbiol. Technol. Engng 3, 277.

PIRT, S. J. (1957). The oxygen requirement of growing cultures of an Aerobacter species determined by means of the continuous culture technique. J. gen. Microbiol. 16, 59.

PirT, S. J. \& Callow, D. S. (1958a). Exocellular product formation by micro-organisms in continuous culture. I. Production of 2:3-butanediol by Aerobacter aerogenes in a single-stage process. J. appl. Bact. 21, 188.

PIRT, S. J. \& CALlow, D. S. (1958b). Observations on foaming and its inhibition in a bacterial culture. J. appl. Bact. 21, 211.

PIRT, S. J. \& CALlow, D. S. (1959). Exocellular product formation by micro-organisms in continuous culture. II. Production of 2:3-butanediol from sucrose by Aerobacter aerogenes in a two-stage process. Sel. scient. Pap. Ist. sup. Sanità 2, 292.

RACKer. E. (1965). In Mechanisms in Bioenergetics. New York: Academic Press. 
SCHARFFER, M. P. (1950). Influence de l'oxygenation des cultures sur la teneur des bactéries en pigments respiratoires à base d'hème. C.r. hebd. Séanc. Acad. Sci. Paris 231, 381.

TEMPEST, D. W. \& Herbert, D. (1965). Effect of dilution rate and growth-limiting substrate on the metabolic activity of Torula utilis cultures. J. gen. Microbiol. 41, 143.

Whrte, D. C. (1963). Factors affecting the affinity for oxygen of cytochrome oxidases in Hemophilus parainfluenzae. J. biol. Chem. 238, 3757.

WINzLER, R. J. (1941). The respiration of bakers' yeast at low oxygen tension. J. cell. comp. Physiol. 17, 263.

Woon, W. A. (1961). Fermentation of carbohydrates and related compounds. In The Bacteria, vol. 2. Ed. I. C. Gunsalus and R. Y. Stainer. New York: Academic Press. 